\title{
Bulk Superconductivity at Ambient Pressure in Polycrystalline Pressed Samples of Organic Metals
}

\author{
D. Schweitzer ${ }^{1}$, S. Kahlich ${ }^{1}$, S. Gärtner ${ }^{2}$, E. Gogu ${ }^{2}$, H. Grimm $^{2}$, R. Zamboni ${ }^{2, *}$, \\ and H.J. Keller ${ }^{3}$
}

13. Physikalisches Institut der Universität Stuttgart, Pfaffenwaldring 57,

D-7000 Stuttgart 80, Fed. Rep. of Germany

2MPI für Medizinische Forschung, AG: Molekülkristalle, Jahnstraße 29,

D-6900 Heidelberg, Fed. Rep. of Germany

${ }^{3}$ Anorganisch Chemisches Institut der Universität Heidelberg,

Im Neuenheimer Feld 270, D-6900 Heidelberg, Fed.Rep.of Germany

* On leave from Istit. di Spectr. Molecolare del C.N.R., Bologna, Italy

Abstract. Bulk superconductivity in polycrystalline pressed samples of $\alpha_{t}-(B E D T-T T F)_{2} I_{3}$ and $\beta_{p}-(B E D T-T T F)_{2} I_{3}$ is reported. This finding shows that organic superconductors can be used in principle for the preparation of electronic devices and superconducting cables.

\section{Introduction}

Organic metals and superconductors usually grow as single crystals at an electrode in an electrochemical cell. Therefore physical investigations of organic metals and superconductors are performed on single crystals. This is certainly an important fact for the understanding of the electronic properties of such materials. On the other hand, in the case of possible applications of such organic metals certainly very rarely single crystals could be used. Since up to now superconducting organic polymers are also not available, the only possible way to use organic superconductors at least in principle for applications would be as polycrystalline powders, which might be pressed to form larger samples.

From a physical point of view superconductivity in such polycrystalline pressed samples should be observable because it has been shown that the coherence lengths in such quasi-two-dimensional organic superconductors are typically of the order of $10-100 \AA$, that means of the order of the dimensions of the unit cell. A problem for the observation of superconductivity in polycrystalline pressed samples might arise from the fact that organic metals are usually relatively soft compared to inorganic superconductors. Therefore the organic materials might undergo phase transitions when a pressure is applied to the powder in order to obtain mechanically stable samples, and the development of annealing processes might be necessary for observing bulk superconductivity.

Here we report the preparation of such polycrystalline pressed samples of organic superconductors which show bulk superconductivity at ambient pressure. 


\section{Experimental and Results}

Mechanically stable samples of the size of $4 \times 1 \times 0.5 \mathrm{~mm}^{3}$ were prepared from carefully pulverized single crystals of organic metals such as $\alpha-(\text { BEDT }- \text { TTF })_{2} I_{3}, \quad u_{t}-(\text { BEDT }- \text { TTF })_{2} I_{3}, \quad \beta-(\text { BEDT }- \text { TTF })_{2} I_{3}$ and $(\mathrm{BEDT}-\mathrm{TTF})_{2} \mathrm{Cu}(\mathrm{NCS})_{2}$ (the crystallites resulting from the pulverisation process had typical diameters of $0.5-10 \mu \mathrm{m}$ ) by applying a pressure of about 1 kbar to the powder. The resistivity of the samples was measured by the usual four point method.

In the case of the polycrystalline pressed samples of (BEDT-TTF) 2 . $\mathrm{Cu}(\mathrm{NS})_{2}$ we were not able to observe bulk superconductivity [1], not even after annealing the samples at $80^{\circ} \mathrm{C}$ for several days. This fact might be due to a phase transition during the preparation of the sample.

In contrast to the samples of (BEDT-TTF) $)_{2} \mathrm{Cu}(\mathrm{NCS})_{2}$ in the case of the polycrystalline pressed samples of $\alpha_{t}-(\text { BEDT -TTF })_{2} I_{3}$, which were prepared from a powder of $\alpha-(\text { BEDT }-T T F)_{2} I_{3}$ and annealed at $75^{\circ} \mathrm{C}$ for at least 3 days directly after the preparation, bulk superconductivity was observed (at $2 \mathrm{~K}$ about $50 \%$ volume superconductivity with respect to an ideal superconductor [1]). The superconducting transition is relatively broad. While the onset for superconductivity in the resistivity curve is found near $9 \mathrm{~K}$, zero resistivity is observed at $2.2 \mathrm{~K}$. In the case of crystals of $\alpha_{t}-(B E D T-T T F)_{2} I_{3}$ zero resistivity appears already at $6 \mathrm{~K}$ [2].

A question which arose was whether it is possible to obtain bulk superconductivity in samples of $\alpha_{t}-(\text { BEDT -TTF })_{2} I_{3}$ when the preparation of the samples starts from $\alpha_{t}-(\text { BEDT }-T T F)_{2} I_{3}$ powder. Curve $a$ in fig. 1 shows the resistivity versus temperature for such a polycrystalline pressed sample of $\alpha_{t}-(\text { BEDT-TTF })_{2} I_{3}$. A metal-like behaviour was found over the whole temperature range between 300 and $1.3 \mathrm{~K}$ but no bulk superconductivity could be found. In contrast after annealing the sample at $75^{\circ} \mathrm{C}$ for 3 days again a broad superconducting transition could be observed (curve,b in fig.1) and ac susceptibility measurements indicate at $2-\mathrm{K}$ a $50 \%$ volume superconductivity with respect to an ideal superconductor. The behaviour of pressed $\alpha_{t}-$ (BEDTTTF) ${ }_{2} I_{3}$ samples prepared in this way was more or less identical with that of samples prepared from $\alpha-(\text { BEDT }-T T F)_{2} I_{3}$ powder [1].

In order to obtain some more information about such phase transitions which occur under pressure during the preparation of the polycrystalline pressed samples, resonance Raman investigations, in particular on the most intensive vibrational symmetric stretching mode of the $\mathrm{I}_{3}^{-}$anions, were carried out. Earlier measurements on single crystals of $\alpha-, \alpha_{t^{-}}$and $\beta-(B E D T-T T F)_{2} I_{3}$ [3] had shown that the resonance Raman-spectra are very sensitive to the symmetry of the $\mathrm{I}_{3}^{-}$anions. The symmetric stretching mode of the linear symmetric $\mathrm{I}_{3}^{-}$ anions is usually found at about $10 \mathrm{~cm}^{-1}$ higher energy than that of the asymmetric and non-linear $\mathrm{I}_{3}^{-}$anions [3]. In the resonance Raman 


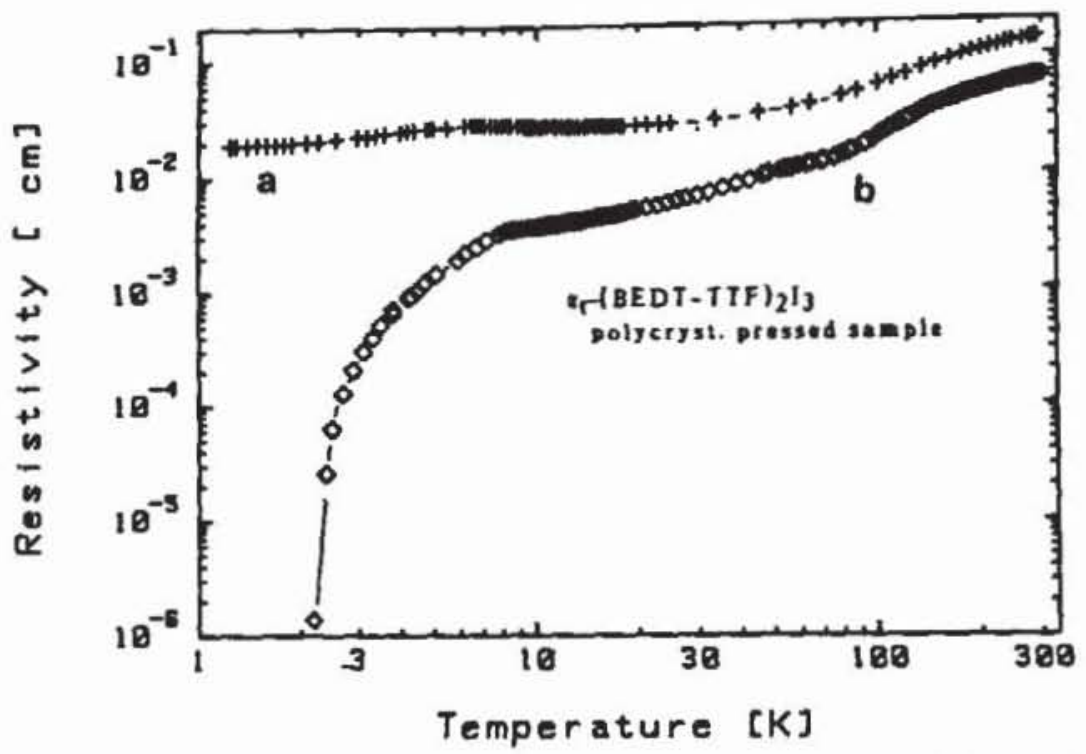

Figure 1: Resistivity versus temperature of a polycrystalline pressed sample of $\alpha_{t}-\left(\right.$ BEDT -TTF) ${ }_{2} 1_{3}$ (curve a) and of a sample of $\alpha_{t}-$ (BEDT TTF) ${ }_{2} I_{3}$ which was annealed after the preparation (curve b).

spectra of the polycrystalline pressed sample of $\alpha-(\text { BEDT }-T T F)_{2} I_{3}$ symmetric, linear and asymmetric $\mathrm{I}_{3}^{-}$anions are observed [4]. This indicates that the pressure during the preparation of the samples deforms the $\mathrm{I}_{3}^{-}$anions partially. A similar result can be observed for the polycrystalline pressed samples which were preprared directly from powdered $\alpha_{t}$-crystals and not annealed after the preparation. In contrast to this finding the resonance Raman spectra of the annealed polycrystalline pressed samples of $\alpha_{t}-(\text { BEDT -TTF) })_{2} I_{3}$ (which become superconducting) show only the stretching mode of the linear and symmetric $\mathrm{I}_{3}^{-}$anions, indicating again the higher symmetry and higher order of the structure.

The most surprising observation was made by measuring the temperature dependence of the resistivity of polycrystalline pressed samples of $\beta-(\text { BEDT }- \text { TTF })_{2} I_{3}$ (in the following called $\beta_{p}-(\text { BEDT-TTF })_{2} I_{3}$ ) [5]. Without annealing the samples showed an onset of superconductivity at $9 \mathrm{~K}$, zero resistivity at $3.2 \mathrm{~K}$ and the middle of the resistive transition at $7.5 \mathrm{~K}$ (see fig. 2). This observation is surprising because single crystals of $\beta-\left(\right.$ BEDT-TTF) ${ }_{2} I_{3}$ show a rather sharp superconducting transition at $1.2 \mathrm{~K} \mathrm{[6]}$ and a metastable superconducting state at $8 \mathrm{~K}$ [7]. Here in the polycrystalline samples of $\beta_{p}-(B E D T-T T F)_{2} I_{3}$ the superconducting state at $7.5 \mathrm{~K}$ is stable and a bulk effect of the sample, as can be seen from the change of the ac susceptibility (see fig. 2 ) which corresponds at $2 \mathrm{~K}$ to about $50 \%$ of that expected for a perfect superconductor.

In the samples of $\beta_{\mathrm{p}}-(\text { BEDT }-\mathrm{TTF})_{2} I_{3}$ a structural phase transition occurring under pressure again plays a role. As a consequence of the phase transition here the transition temperature into the superconducting state is increased. This behaviour reemphasizes that organic superconductors might also be of interest for industrial applications. 


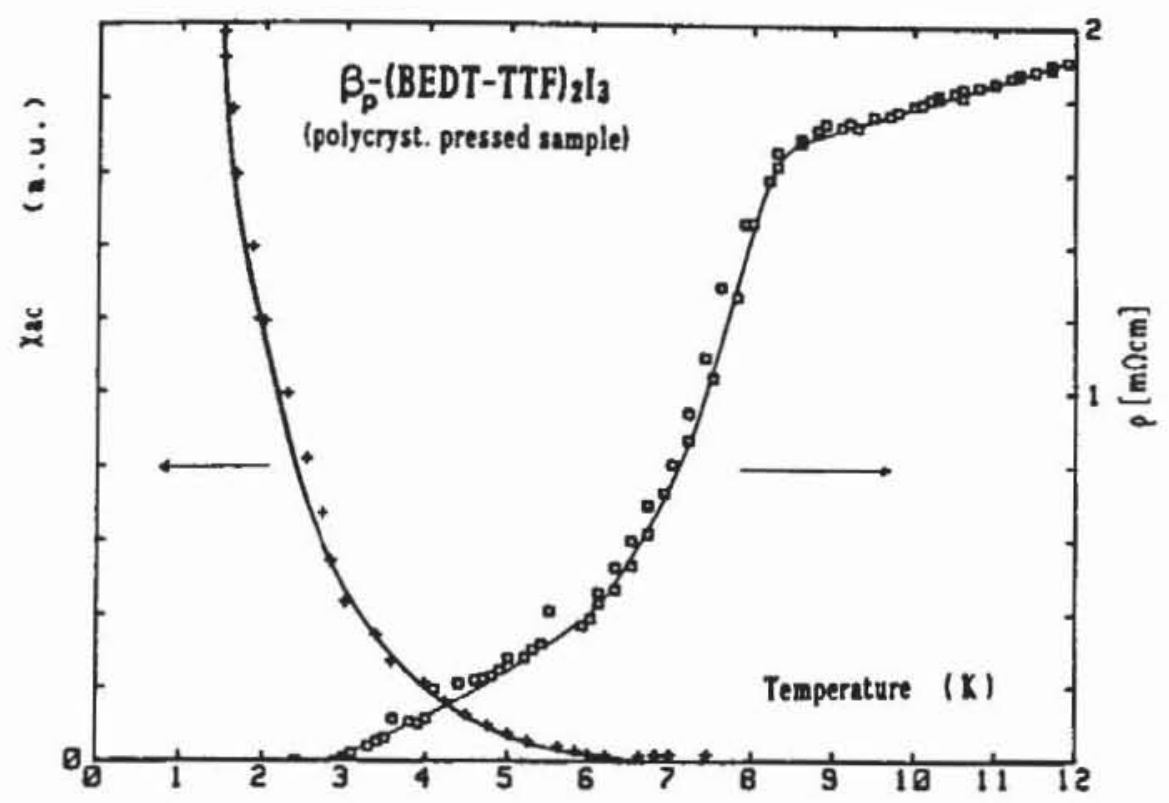

Figure2: Resistivity and change in ac-susceptibility versus temperature of a polycrystalline sample of $\beta_{\mathrm{p}}-(\text { BEDT }-\mathrm{TTF})_{2} \mathrm{I}_{3}$ (below $12 \mathrm{~K}$ ).

\section{References.}

[1] D. Schweitzer et al. Solid State Comm. 69, 843 (1989).

[2] D. Schweitzer et al. Z. Phys. B - Cond. Matt. 67, 489 (1987).

[3] R. Swietlik et al., Phys. Rev. B. 36, 6881 (1987).

[4] R. Zamboni et al., in "Lower Dimensional Systems and Molecular Electronics", Plenum Press 1989, in print.

[5] D. Schweitzer et al., Angew. Chem. Adv. Mater. 101, 977 (1989).

[6] E. B. Yagubskii et al., Sov. Phys. JETP Lett. 39,12 (1984).

[7] F. Creuzet et al., J. Phys. (Paris) Lett. 46, L-1079 (1985). 